\title{
ON THE ABSENCE OF NECESSARY CONDITIONS \\ FOR LINEAR EVOLUTION OPERATORS
}

\author{
JEROME A. GOLDSTEIN 1
}

\begin{abstract}
There exist selfadjoint operators $A(t)(t>0)$, whose resolvents depend smoothly on $t$, such that the initial value (Schrödinger) problem $d u(t) / d t=i A(t) u(t), u(0)=f$ has a unique $\left(C^{\infty}\right)$ solution $u$ for each $f$ in a dense set in the underlying Hilbert space, with $u$ depending continuously on $f$, and yet the intersection over $t \geqslant 0$ of the domain of $A(t)$ is $\{0\}$.
\end{abstract}

1. Introduction. Many authors have studied the linear evolution equation Cauchy problem

$$
\begin{gathered}
d u(t) / d t=B(t) u(t) \quad(t \geqslant 0), \\
u(0)=f
\end{gathered}
$$

in a Banach space $X$. Roughly speaking, if for each $t, B(t)$ is $m$-dissipative (i.e. $B(t)$ generates a $\left(C_{0}\right)$ contraction semigroup on $X$ (cf. [5])), if the domain $\mathscr{D}(B(t))$ of $B(t)$ does not vary too wildly with $t$, and if $B(t)$ depends on $t$ smoothly in a suitable sense, then (1), (2) has a unique solution $u$ for all $f$ in some dense set, with $u$ depending continuously on $f$. See, for instance, Kato [6], [7], Darmois [1], Dorroh [2], and the references cited therein. In all the well-posedness studies concerning (1), (2), the sufficient conditions for solvability are such that $\cap\{\mathscr{D}(B(t)): t \geqslant 0\}$ is dense in $X$.

The purpose of this note is to construct operators $B(t)=i A(t)$ satisfying the following four conditions.

(i) For each $t \geqslant 0, A(t)$ is a nonnegative selfadjoint operator on a complex Hilbert space $H$ (hence each $B(t)=i A(t)$ generates a $\left(C_{0}\right)$ unitary group on $H$ ).

(ii) $A(t)$ depends smoothly on $t$ in the sense that for each complex number $\lambda \notin[0, \infty)$ and each $f \in H, t \rightarrow(\lambda I-A(t))^{-1} f$ is in $C^{\infty}([0, \infty), H)$.

(iii) For each $f$ in a dense set $D \subset H$, the problem (1), (2) has a unique solution $u \in C^{\infty}([0, \infty), H)$ which depends continuously on $f$.

(iv) $\cap\{\mathscr{D}(A(t)): t \geqslant 0\}=\{0\}$.

Received by the editors August 1, 1976 and, in revised form, November 22, 1976.

AMS (MOS) subject classifications (1970). Primary 47D99; Secondary 34G05, 47B44.

1 Partially supported by N.S.F. grant MCS76-06515. 
When $B(t) \equiv B$ is time independent, then the problem (1), (2) is wellposed if and only if $B$ generates a $\left(C_{0}\right)$ semigroup [10], in which case $\mathscr{D}(B)$ is necessarily dense according to the Hille-Yosida theorem [5], [11]. Our example thus illustrates a fundamental difference between the Cauchy problem for (1) when $B$ depends on $t$ and when $B$ does not, i.e., between evolution operator theory and semigroup theory. As mentioned above, in the time independent case there are conditions which are simultaneously necessary and sufficient for the well-posedness of (1), (2). In the time dependent case, there are definitive sufficient conditions [6], [7], but this is all. Our example shows that necessary conditions must be weaker than the sufficient conditions in the time dependent case.

Of course, if one makes essential restrictions on the class of families $\{B(t)\}$ (or equivalently, the class of equations (1)) that one considers, then it becomes possible to obtain necessary and sufficent conditions in order that a family $\{B(t)\}$ generate an evolution operator family $\{U(t, s)\}$ governing (1). The first result along these lines was obtained by Kömura [8]. Lovelady [9] has worked on a related problem. Our example answers the question raised in the review of Kömura's article [4].

2. The example. Let $V$ be a nonnegative selfadjoint operator on a separable infinite-dimensional complex Hilbert space $H$. Let $\chi$ be a nonnegative $C^{\infty}$ function on $[0, \infty)$ with $\chi \neq 0$. Let

$$
Z(t) g=\exp \left(i \int_{0}^{t} \chi(s) d s V\right) g
$$

for $g \in H$. If $g \in \mathscr{D}^{\infty}(V) \equiv \cap_{n=1}^{\infty} \mathscr{D}\left(V^{n}\right)$, then $Z(\cdot) g \in C^{\infty}([0, \infty), H)$. Recall that $\mathscr{D}^{\infty}(V)$ is dense in $H[5$, p. 308].

Now let $S$ be any unbounded nonnegative operator on $H$. According to a theorem of von Neumann [3, p. 273], there is a unitary operator $U_{1}$ on $H$ such that the nonnegative operator $T \equiv U_{1} S U_{1}^{*}$ satisfies $\mathscr{D}(T) \cap \mathscr{D}(S)=\{0\}$. Since $T^{n}=U_{1} S^{n} U_{1}^{*}$ for $n=1,2,3, \ldots$ it follows that $U_{1}$ is a bijection from $\mathscr{Q}^{\infty}(S)$ to $\mathscr{D}^{\infty}(T)$.

Represent $U_{1}$ by the spectral theorem as $U_{1}=\int_{0}^{2 \pi} e^{i \lambda} d E_{\lambda}$. Defining $L$ $=\int_{0}^{2 \pi} \lambda d E_{\lambda}$, we have that $L$ is a bounded nonnegative selfadjoint operator on $H$ and $\{U(t)=\exp (i t L): t$ real $\}$ is a $\left(C_{0}\right)$ unitary group with $U(1)=U_{1}$.

We are now ready to construct the example. Let $\phi, \eta, \psi$ be nonnegative nontrivial $C^{\infty}$ functions on $[0, \infty)$ satisfying supp $\phi \subset[0,1)$, supp $\eta \subset(1,2)$, supp $\psi \subset(2,3)$, and $\int_{0}^{2} \eta(s) d s=1$. Let

$$
A(t)=\phi(t) S+\eta(t) L+\psi(t) T, \quad t \text { real, }
$$

let $B(t)=i A(t)$, and let $D=\mathscr{D}^{\infty}(S)$, which is dense in $H$. For $f \in D$ the Cauchy problem (1), (2) has a unique solution given by 


$$
u(t)= \begin{cases}\exp \left(i \int_{0}^{t} \phi(s) d s S\right) f & \text { for } 0 \leqslant t \leqslant 1 \\ \exp \left(i \int_{0}^{t} \eta(s) d s L\right) u(1) & \text { for } 1 \leqslant t \leqslant 2, \\ \exp \left(i \int_{0}^{t} \psi(s) d s T\right) u(2) & \text { for } 2 \leqslant t<\infty .\end{cases}
$$

To see this we apply the discussion in the first paragraph of this section, taking successively the triple $\{V, \chi(t), g\}$ to be $\{S, \phi(t), f\},\{L, \eta(t-1), u(1)\}$, and $\{T, \psi(t-2), u(2)\}$. First of all, $u \in C^{\infty}([0,1], H)$ and $u$ satisfies (1), (2) on $[0,1]$, since $f \in \mathscr{D}^{\infty}(S)$. Since $u(1) \in \mathscr{D}^{\infty}(L)=H$, it follows that

$$
u \in C^{\infty}([1,2], H)
$$

and (1) holds on [1, 2]. Next,

$$
u(2)=\exp \left(i \int_{0}^{2} \eta(s) d x L\right) u(1)=U(1) u(1)=U_{1} u(1) .
$$

Moreover,

$$
u(1)=\exp \left(i \int_{0}^{1} \psi(s) d s S\right) f \in \mathscr{D}^{\infty}(S)
$$

since $e^{i t S}$ leaves $\mathscr{D}^{\infty}(S)$ invariant. Consequently, $u(2)=U_{1} u(1) \in \mathscr{D}^{\infty}(T)$, and it follows that $u \in C^{\infty}([2, \infty) ; H)$ and (1) holds on $[2, \infty)$. Since $u(t)$ is constant near $t=1$ and near $t=2$, the one-sided derivatives match up at 1 , 2 , and so $u$ defined by (3) satisfies

( $\alpha) u \in C^{\infty}([0, \infty), H)$ for each $f \in \mathscr{D}$;

( $\beta) u$ satisfies (1), (2);

$(\gamma) u$ depends continuously on $f$.

Thus (i), (iii), (iv) hold. That (ii) also holds follows easily from the fact that

$$
(\lambda I-A(t))^{-1}=(\lambda I+\phi(t) S+\eta(t) L+\psi(t) T)^{-1}
$$

which equals either

$$
\lambda^{-1} I \text { or }(\lambda I+\phi(t) S)^{-1} \text { or }(\lambda I+\eta(t) L)^{-1} \text { or }(\lambda I+\psi(t) T)^{-1} \text {. }
$$

The verification of (i)-(iv) is thus complete.

3. One can take $D=H$. We can allow $D$ to be $H$ if we seek a solution $u \in C^{\infty}((0, \infty), H) \cap C([0, \infty), H)$ satisfying (1) on $(0, \infty)$ and (2). This notion is often used in discussions of parabolic problems. To obtain the required example, let $B(t)$ be as before on $[1, \infty)$, and on $[0,1]$ instead of $B(t)=i \phi(t) S$ take $B(t)=-\phi(t) S$ and assume further that $\phi(0)>0$. Then $u(t)=\exp \left(-\int_{0}^{t} \phi(s) d s S\right) f$ satisfies $u \in C^{\infty}((0,1], H) \cap C([0,1], H)$ for each $f \in H$. This follows easily from the operational calculus associated with the spectral theorem; alternatively it also follows from the theory of analytic semigroups. Necessarily $u(1)$ will be in $\mathscr{D}^{\infty}(S)$, and the desired result is a 
consequence of the computations in the previous section.

We are grateful to J. R. Dorroh for his helpful comments on the first draft of this paper.

\section{REFERENCES}

1. G. F. Darmois, Evolution equations in a Banach space, Ph.D. Thesis, Univ. of California, Berkeley, 1975.

2. J. R. Dorroh, A simplified proof of a theorem of Kato on linear evolution equations, J. Math. Soc. Japan 27 (1975), 474-478.

3. P. A. Fillmore and J. P. Williams, On operator ranges, Advances in Math. 7 (1971), 254-281.

4. J. A. Goldstein, Review of Kömura's paper, [8]. MR 44 \#4567.

5. E. Hille and R. S. Phillips, Function analysis and semi-groups, Amer. Math. Soc. Colloq. Publ., vol. 31, Amer. Math. Soc., Providence, R.I., 1957.

6. T. Kato, Linear evolution equations of "hyperbolic" type, J. Fac. Sci. Univ. Tokyo Sect. IA 17 (1970), 241-258.

7. , Linear evolution equations of "hyperbolic" type. II, J. Math. Soc. Japan 25 (1973), 648-666.

8. Y. Kömura, On linear evolution operators in reflexive Banach spaces, J. Fac. Sci. Univ. Tokyo Sect. IA 17 (1970), 529-542.

9. D. L. Lovelady, On the generation of linear evolution operators (to appear).

10. R. S. Phillips, A note on the abstract Cauchy problem, Proc. Nat. Acad. Sci. U.S.A. 40 (1954), 244-248.

11. K. Yosida, Functional analysis, Springer, Berlin, 1965.

Department of Mathematics, Tulane University, New Orleans, Louisiana 70118 